\title{
Confiabilidad interevaluador de la prueba video head impulse test en estudiantes de primer año de la generación 2016 de la Escuela de Fonoaudiología de la Universidad de Valparaíso Casa Central
}

\author{
Inter-rater reliability of the video head impulse test in students from the \\ School of Speech and Language therapist of the University of \\ Valparaiso main campus class 2016
}

Andre Gómez L1', Valeria Álvarez N², Allison López $\mathrm{R}^{2}$, Rodrigo Neira $\mathbf{M}^{2}$.

\begin{abstract}
RESUMEN
Introducción: Elvideo head impulse test es un método rápido, no invasivo y cómodo para evaluar el reflejo vestíbulo ocular, el cual está siendo utilizado con mayor frecuencia en la evaluación de pacientes con alteraciones del equilibrio. Sin embargo, en Chile se evidencia una falta de estudios relacionados a la confiabilidad interevaluador de dicha prueba.

Objetivo: Determinar el nivel de concordancia de los resultados de la eficiencia del reflejo vestíbulo ocular de los canales semicirculares horizontales, obtenidos mediante la aplicación de la prueba video head impulse test, por parte de 3 evaluadores.

Material y método: Estudio cuantitativo, correlacional, no experimental transversal descriptivo, con muestreo no probabilístico, de participación voluntaria. La prueba video head impulse test fue aplicada por 3 examinadores a 30 estudiantes que cumplieron con los criterios de inclusión.

Resultados: El método de Bland y Altman mostró gran concordancia en las mediciones realizadas entre los 3 examinadores. La correlación intraclase para la ganancia del canal semicircular horizontal derecho e izquierdo fue de 0,85 y 0,91 respectivamente y de 0,75 para el valor de asimetría.

Conclusión: El estudio mostró una confiabilidad interevaluador adecuada de la prueba video head impulse test para la evaluación de los canales semicirculares horizontales.

Palabras clave: Video head impulse test, reflejo vestíbulo ocular, confiabilidad interevaluador.
\end{abstract}

\section{ABSTRACT}

Introduction: The video head impulse test is a rapid, non-invasive and comfortable method for the evaluation of the vestibulo-ocular reflex that is being used more often

1 Fonoaudiólogo, Laboratorio de Audiología y Vestibular, Escuela de Fonoaudiología Casa Central, Facultad de Medicina, Universidad de Valparaíso, Viña del Mar, Chile.

2 Fonoaudiólogo, Universidad de Valparaíso, Chile.

Recibido el 13 de noviembre, 2017. Aceptado el 11 de diciembre, 2017. 
in the evaluation of patients with balance disorders. In Chile there is a lack of studies related to the inter-rater reliability for this test.

Aim: To determine the level of agreement on the results for the vestibulo-ocular reflex of the horizontal semicircular canals obtained by the execution of the video head impulse test by 3 raters.

Material and method: Quantitative, correlational, non-experimental, cross-sectional descriptive study with a type of non-probability sampling with voluntary participation. The video head impulse test was applied by 3 raters to 30 students who fulfilled the inclusion criteria.

Results: The Bland-Altman method demonstrated good agreement in the measurements made between the 3 raters. The inter-rater reliability for the gain of the left and right horizontal semicircular canals was 0.85 and 0.91 respectively and 0.75 for the asymmetry value.

Conclusion: The study showed an adequate inter-rater reliability for the video head impulse test in the evaluation of the horizontal semicircular canals.

Key words: vHIT, vestibular ocular reflex, inter-rater reliability.

\section{INTRODUCCIÓN}

La exploración del reflejo vestíbulo ocular (RVO) es una práctica clínica de gran importancia en la evaluación de pacientes con alteración del equilibrio ${ }^{1,2}$. Entre las herramientas que pueden ser utilizadas para la evaluación del RVO se encuentra la prueba video head impulse test (v-HIT) ${ }^{3}$, un método cómodo y poco invasivo para el usuario ${ }^{4}$, capaz de detectar de forma rápida y objetiva alteraciones en el funcionamiento del RV05. Este método tiene la ventaja de registrar alteraciones sutiles del RVO, alcanzando una especificidad del $93 \%$ y una sensibilidad del $74 \%{ }^{6}$.

Los parámetros obtenidos por la prueba v-HIT para evaluar alteraciones del RVO en el plano horizontal son el promedio de ganancia de los impulsos cefálicos realizados hacia derecha e izquierda, el porcentaje promedio de asimetría entre ellos y la presencia de sacadas correctivas, cubiertas y descubiertas. Los valores normativos de ganancia en esta prueba deben ser cercanos a 1 en sujetos sanos ${ }^{4}$, incluso para adultos mayores, como lo demuestra un estudio normativo internacional para los valores de ganancia de todos los canales semicirculares según $\operatorname{edad}^{7}$. En Chile, una investigación realizada en una población de estudiantes universitarios sanos, obtuvo resultados promedio de ganancia para impulsos cefálicos a derecha e izquierda de 0,92 y 0,85 respectivamente, una asimetría promedio de $10 \%$ y la ausencia de sacadas cubiertas y descubiertas ${ }^{8}$.
Si bien, los estudios anteriores ${ }^{7,8}$ muestran que la prueba v-HIT es eficiente, no existe evidencia acerca de la variabilidad interevaluador que pudiese generarse en este examen, principalmente debido a la activa participación del examinador, donde éste debe tomar la cabeza del paciente y realizar impulsos cefálicos similares a los de head impulse test, con velocidades entre los $150 \%$ y $250^{\circ} / \mathrm{s}^{9}$. Así, se hace necesario determinar la confiabilidad interevaluador de la prueba v-HIT, ya que, la práctica clínica, independiente de la especialidad, requiere el uso de mediciones confiables, evitando que las intervenciones y los resultados se vean comprometidos, por mediciones subjetivas ${ }^{10}$. Para que un instrumento sea considerado confiable, debe responder de forma fidedigna, secuencial, exacta, predecible y sin cambios, incluso al ser aplicada por distintos evaluadores ${ }^{11,12}$.

Para determinar la confiabilidad interevaluador de la prueba v-HIT se buscará obtener el nivel de concordancia existente entre los resultados obtenidos por 3 evaluadores diferentes. El nivel de concordancia permite medir cuán acordes son entre sí, distintas observaciones sobre mismo evento ${ }^{12}$. En el caso de este estudio se desea medir el nivel de acuerdo entre las mediciones realizadas con la prueba v-HIT, por 3 evaluadores diferentes sobre un mismo sujeto.

Para medir el nivel de concordancia en pruebas clínicas, se han propuesto distintos métodos ${ }^{13}$; estos van a depender del tipo de variable que se 
desee medir. En el caso de este estudio, en que fueron medidas variables cuantitativas continuas, se utilizó el coeficiente de correlación intraclase ${ }^{14} y$ el método propuesto por Bland y Altman ${ }^{15}$.

\section{OBJETIVO}

El objetivo de esta investigación es determinar el nivel de concordancia de los resultados sobre la eficiencia del RVO de los canales semicirculares horizontales, obtenidos mediante la aplicación de la prueba v-HIT por 3 evaluadores distintos, en estudiantes de primer año de la Escuela de Fonoaudiología de la Universidad de Valparaíso, Casa Central. De esta forma, se contará con información valiosa acerca de la confiabilidad interevaluador de esta prueba, que actualmente se ha transformado en una herramienta fundamental en el área de la otoneurología.

\section{MATERIAL Y MÉTODO}

Se realizó un estudio de tipo cuantitativo, con un alcance correlacional y un diseño no experimental transversal descriptivo. El tipo de muestreo fue no probabilístico, con una muestra inicial conformada por 34 estudiantes voluntarios de primer año matriculados en 2016 en la Escuela de Fonoaudiología de Universidad de Valparaíso, Casa Central. Todos los participantes cumplieron con los criterios de inclusión que apuntaban a ausencia de patología auditiva y vestibular, sin embargo, 4 estudiantes fueron excluidos por presentar tensión cervical exacerbada; de esta forma, la muestra final quedó conformada por 30 estudiantes. La edad de los participantes fluctuó entre 18 y 22 años (con promedio de 18,33 años y desviación estándar de 0,99 años), dividiéndose en 2 hombres y 28 mujeres.

En cuanto al procedimiento, luego de haber recibido el acta de aprobación del Comité de Bioética de la Facultad de Medicina de la Universidad de Valparaíso, se entregó la información pertinente a cada estudiante, de manera verbal y escrita. Cada participante que se interesó debió leer el consentimiento informado y firmar el acta correspondiente. La selección de la muestra, se llevó a cabo utilizan- do criterios de inclusión y exclusión, para lo cual se aplicó una anamnesis auditiva-vestibular, una otoscopía y una audiometría de barrido. Luego, a los estudiantes que cumplieran con los criterios de inclusión se les realizó la prueba v-HIT con el instrumento e-HITUSB de la casa comercial BioMed Jena, en los laboratorios de audiología y vestibular de la Escuela de Fonoaudiología de la Universidad de Valparaíso.

Para llevar a cabo la prueba, uno de los investigadores (no evaluador), le solicitó al estudiante tomar asiento en una silla ubicada a 1 metro de distancia de la pared, luego le acomodó las gafas de video-oculografía y calibró el instrumento. A continuación, se le indicó al participante que era necesario mantener fija su mirada en un punto central ubicado frente a él, idealmente sin pestañear, con la cabeza inclinada $30^{\circ}$ en el plano vertical inferior. Una vez cumplidos los pasos descritos anteriormente y luego de haber calibrado la prueba, el primer evaluador se ubicó detrás del estudiante sujetando su cabeza con ambas manos; ejecutando 15 movimientos hacia la derecha e izquierda de manera rápida, brusca e impredecible, en un rango entre $15^{\circ}-25^{\circ}$, con una velocidad sobre los $150^{\circ} / \mathrm{s}$, retornando al punto central entre cada rotación. El rango y la velocidad del movimiento se pudieron medir gracias a la retroalimentación visual que entregó el equipo. Así, el examinador tuvo información en tiempo real de tales parámetros, asegurándose del cumplimiento de éstos.

Cabe destacar que, para la medición de la muestra, los 3 evaluadores llevaron a cabo la prueba de forma ciega frente a la aplicación previa. El orden de ingreso de cada examinador, para evaluar a cada estudiante, fue al azar; es decir, ingresó uno de los 3 evaluadores escogidos aleatoriamente, realizó la medición y se retiró del box. A continuación, se repitió el mismo procedimiento con el segundo y tercer evaluador, para luego reiterar la práctica hasta completar el total de la muestra. Es importante mencionar, que las mediciones fueron realizadas en varias jornadas para evitar la fatiga de los examinadores y que entre cada una ellas existió una pausa, para así evitar el cansancio de los examinados. De todas formas, frente a la consulta, ninguno de ellos refirió molestia o cansancio frente al procedimiento.

Los resultados fueron consignados en una base de datos y procesados mediante el uso de los 
programas computacionales Excel versión 2013, SPSS Statistics versión 23 y MedCalc versión 17. Se comenzó con un análisis descriptivo de las variables mediante tablas con distintas estadísticas y luego se realizó el contraste de normalidad de las variables. Finalmente, para el análisis de concordancia de los resultados obtenidos entre los distintos evaluadores, se utilizó el método de Bland y Altman y la prueba de correlación intraclase para muestras independientes, con una probabilidad de error del $5 \%$.

\section{RESULTADOS}

Los resultados de la investigación se presentan para las variables: ganancia del canal semicircular horizontal derecho (ganancia del CSCHD), ganancia del canal semicircular horizontal izquierdo (ganancia del CSCHI) y asimetría entre ambas ganancias; cada una de ellas para los 3 evaluadores.

Para realizar el contraste de normalidad, se aplicó la prueba Shapiro-Wilk, resultando las 3 variables normalmente distribuidas, con un valor $p>0,05$. En la Tabla 1, se puede observar que los valores promedios de la ganancia del CSCHD, para los 3 evaluadores, fluctúan entre 0,93 y 0,96, con una desviación estándar entre 0,08 y 0,09; en cambio, para la ganancia del CSCHI, los promedios varían entre 0,89 y 0,97 , presentando un rango mayor que el de los promedios de las ganancias del CSCHD, con una desviación estándar entre 0,09 y 0,10 . Respecto a la asimetría, el valor promedio va desde $-0,85 \%$ a $3,74 \%$, con una desviación estándar de $3,05 \%$ a $4,12 \%$. Es importante mencionar que para todas las variables (ganancia del CSCHD, ganancia del CSCHI y asimetría) de los 3 examinadores, existe una diferencia mínima entre la media y la mediana, lo cual habla de distribuciones simétricas.

En las Figuras 1, 2 y 3 (gráficos de Bland y Altman), es posible observar un diagrama de puntos de la diferencia entre las mediciones realizadas a los 30 participantes del estudio por las parejas de evaluadores $1-2,1-3$ y $2-3$ contra su promedio para las 3 variables del estudio. Sobre este diagrama se aprecia su diferencia media (representada por la línea ennegrecida) y los límites de concordancia (líneas discontinuas).

Las diferencias medias (sesgo) entre las distintas parejas de evaluadores para las variables: ganancia del CSCHD (Figura 1), ganancia del CSCHI (Figura 2) y asimetría (Figura 3) se encuentran muy cercanas a 0 y no son clínicamente significativas (Tabla 2).

Además, los puntos del diagrama se encuentran distribuidos en $90 \%$ o más, a uno y otro lado de las diferencias medias, dentro de los límites de concordancia, para las 3 parejas de evaluadores, reflejando gran acuerdo entre las evaluaciones realizadas por los 3 examinadores.

Con el fin de obtener el índice de concordancia entre los 3 examinadores para las variables ganancia del CSCHD, ganancia del CSCHI y asimetría, se calculó el coeficiente de correlación intraclase (CCI) para mediciones promediadas (ya que cada

Tabla 1. Medidas descriptivas de las variables: ganancia del CSCHD, ganancia del CSCHI y asimetría, respecto a los tres evaluadores

\begin{tabular}{|l|lllc|}
\hline \multicolumn{2}{|c}{} & Promedio & DE & Mediana \\
\hline \multirow{2}{*}{ Evaluador 1} & Ganancia del CSCHD & 0,96 & 0,09 & 0,94 \\
& Ganancia del CSCHI & 0,89 & 0,10 & 0,89 \\
& Asimetría & $3,74 \%$ & $3,05 \%$ & $3,75 \%$ \\
\hline \multirow{2}{*}{ Evaluador 2 } & Ganancia del CSCHD & 0,96 & 0,08 & 0,95 \\
& Ganancia del CSCHI & 0,97 & 0,09 & 0,99 \\
& Asimetría & $-0,85 \%$ & $3,08 \%$ & $-0,50 \%$ \\
\hline \multirow{2}{*}{ Evaluador 3 } & Ganancia del CSCHD & 0,93 & 0,08 & 0,95 \\
& Ganancia del CSCHI & 0,92 & 0,10 & 0,91 \\
& Asimetría & $0,59 \%$ & $4,12 \%$ & $0,30 \%$ \\
\hline
\end{tabular}




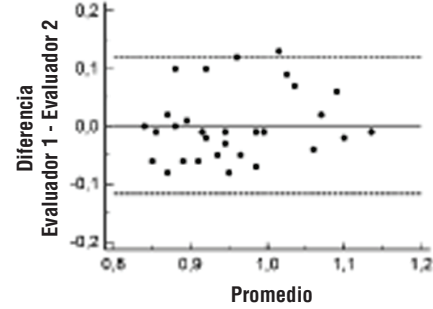

Evaluador 1 - Evaluador 2

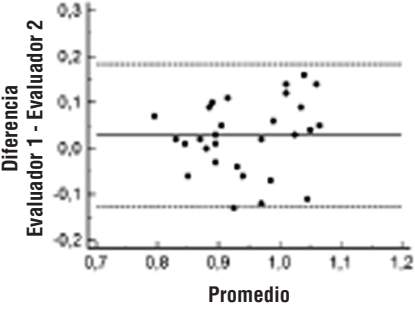

Evaluador 1 - Evaluador 3

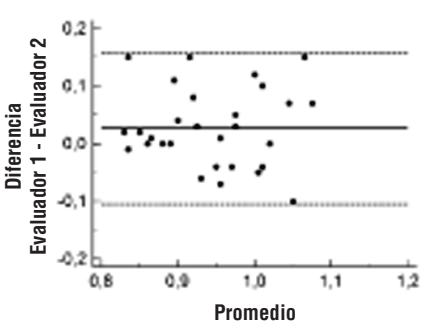

Evaluador 2 - Evaluador 3

Figura 1. Gráfico de Bland y Altman para la variable ganancia del CSCHD para las parejas de evaluadores 1-2, 1-3 y 2-3.

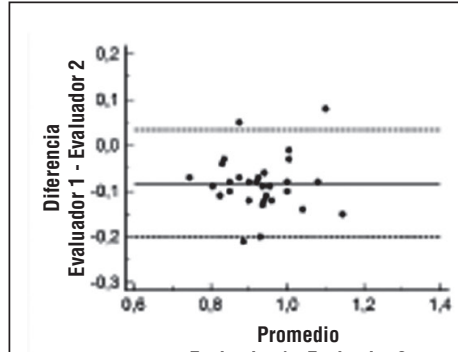

Evaluador 1 - Evaluador 2

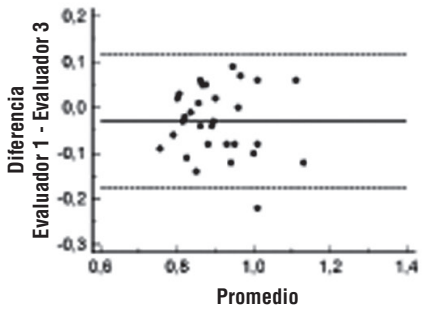

Evaluador 1 - Evaluador 3

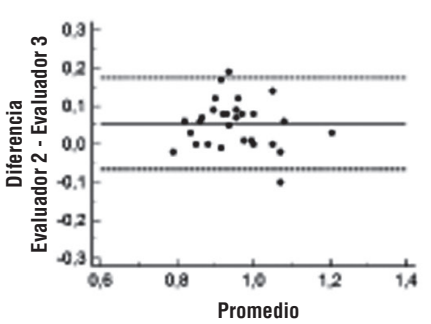

Evaluador 2 - Evaluador 3

Figura 2. Gráfico de Bland y Altman para la variable ganancia del CSCHI para las parejas de evaluadores 1-2, 1-3 y 2-3.
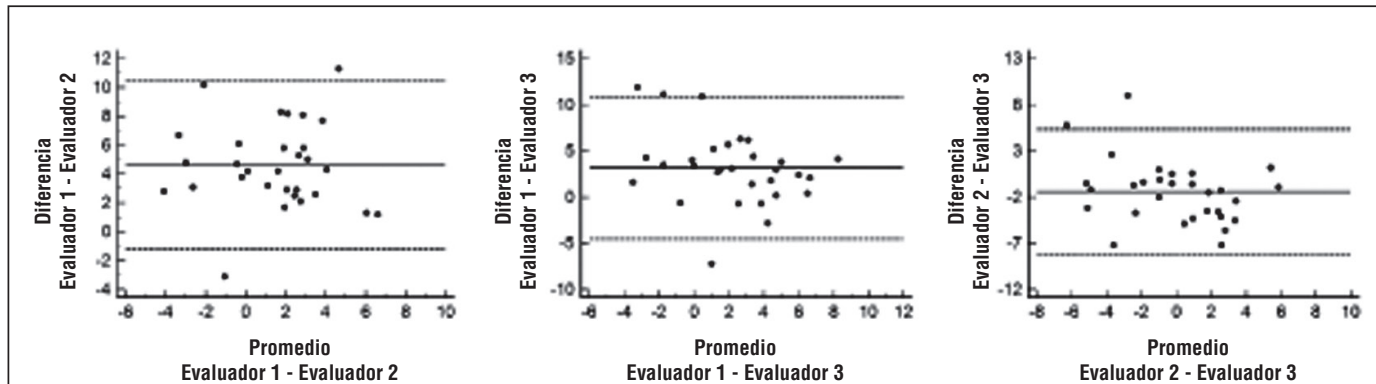

Figura 3. Gráfico de Bland y Altman para la variable asimetría para las parejas de evaluadores 1-2, 1-3 y 2-3.

Tabla 2. Medidas descriptivas de los valores de concordancia de ganancia del CSCHD, ganancia del CSCHI y asimetría entre evaluadores 1-2, evaluadores 1-3, y evaluadores 2-3

\begin{tabular}{|l|cccccc|}
\cline { 6 - 7 } & \multicolumn{1}{c}{} & & $95 \%$ intervalo de confianza de la diferencia \\
\hline \multirow{2}{*}{ Ganancia del CSCHD } & Evaluadores & $\mathrm{n}$ & Diferencia media & DE & Inferior & Superior \\
& 1 y 2 & 30 & 0,0013 & 0,06050 & $-0,0213$ & 0,0239 \\
& 1 y 3 & 30 & 0,0280 & 0,07885 & $-0,0014$ & 0,0574 \\
Ganancia del CSCHI & 2 y 3 & 30 & 0,0267 & 0,06738 & 0,0015 & 0,0518 \\
& 1 y 2 & 30 & $-0,0843$ & 0,05992 & $-0,1067$ & $-0,0620$ \\
Asimetría & 1 y 3 & 30 & $-0,0310$ & 0,07508 & $-0,0590$ & $-0,0030$ \\
& 2 y 3 & 30 & 0,0533 & 0,06144 & $-0,0304$ & 0,0763 \\
& 1 y 2 & 30 & 4,5900 & 2,960000 & 3,4847 & 5,6953 \\
& 1 y 3 & 30 & 3,1500 & 3,92417 & 1,6847 & 4,6153 \\
& 2 y 3 & 30 & $-1,4400$ & 3,44139 & $-2,7250$ & $-1,1550$ \\
\hline
\end{tabular}


valor informado corresponde al promedio de 15 mediciones). En la Tabla 3, es posible apreciar un CCI de 0,85 , con un intervalo de confianza de $95 \%$ de 0,73 a 0,93 para la ganancia del CSCHD. En el caso de la ganancia del CSCHI, se observa un $\mathrm{CCl}$ de 0,91 , con un intervalo de confianza de $95 \%$ de 0,84 a 0,95 .

Finalmente, el índice de concordancia de la variable de asimetría, se puede evidenciar a través de un CCI de 0,75 con un intervalo de confianza de $95 \%$ de 0,54 a 0,87 . Cada $\mathrm{CCI}$ para las 3 variables de estudio obtuvo un valor $p<0,001$.

Es importante destacar que frente a la determinación de la presencia de sacadas correctivas, por parte de los 3 examinadores, se obtuvo un acuerdo total, donde ninguno de ellos registró la presencia de sacadas cubiertas ni descubiertas en toda la investigación.

\section{DISCUSIÓN}

En cuanto a los valores obtenidos por los 3 examinadores para la ganancia del CSCHD y ganancia del CSCHI, se puede mencionar que éstos concuerdan con los valores normativos obtenidos por otros estudios 7,8 , tomando como referencia de normalidad, valores cercanos a uno. De igual forma, los valores de asimetría se encuentran dentro de un rango normal cercano a cero ${ }^{16}$. Con respecto a la concordancia observada entre las mediciones realizadas por las distintas parejas de evaluadores para las variables ganancia del CSCHD, ganancia del CSCHI y asimetría se puede afirmar que existe un alto nivel de concordancia debido a los siguientes puntos:
- En todos los casos, la diferencia media entre evaluadores fue mínima (Tabla 2), siendo ésta clínicamente irrelevante.

- Los límites de concordancia son lo suficientemente acotados en todos los casos, asegurando mediciones clínicamente concordantes.

- Las diferencias de las mediciones realizadas a todos los participantes del estudio, se encuentran distribuidas en $90 \%$ más, dentro de los límites de concordancia.

- No se observan errores sistemáticos, ya que las diferencias entre las parejas se distribuyen a ambos lados de la media de las diferencias.

Los resultados obtenidos en el cálculo del CCI, para las mediciones realizadas por los 3 examinadores (Tabla 3), muestran buena confiabilidad interevaluador para las variables ganancia del CSCHD y asimetría; y excelente para la variable ganancia del CSCHI de acuerdo con los valores de referencia para el CCl14.

Es importante mencionar que, el acuerdo total que existió entre los 3 examinadores en determinar la ausencia de sacadas correctivas, en todos los participantes de esta investigación, coincide con los resultados obtenidos por otro estudio ${ }^{8}$ que trabajó con sujetos jóvenes y sanos. Además, la presencia de sacadas correctivas, ya sea cubiertas 0 descubiertas, reflejarían alteraciones en el $R V 0^{4,6,16}$, lo cual no aplica en este caso, ya que se trabajó con sujetos jóvenes y sanos.

Cabe destacar que, actualmente a nivel nacional no se han registrado estudios que evalúen la confiabilidad interevaluador de la prueba v-HIT. Para los investigadores, la alta confiabilidad de los resultados obtenidos, pudo estar relacionada con

Tabla 3. Coeficiente de correlación intraclase de los valores promedio de ganancia del CSCHD, ganancia del CSCHI y asimetría, entre los tres evaluadores

\begin{tabular}{|l|c|cc|cccc|}
\hline \multicolumn{2}{|c|}{} & \multicolumn{2}{|c|}{$95 \%$ Intervalo de confianza } & \multicolumn{4}{c|}{ Prueba F con valor verdadero 0 } \\
\hline Variable & $\begin{array}{c}\text { Correlación } \\
\text { intraclase }\end{array}$ & $\begin{array}{c}\text { Límite } \\
\text { inferior }\end{array}$ & $\begin{array}{c}\text { Límite } \\
\text { superior }\end{array}$ & Valor & gl1 & gl2 & Sig. \\
\hline Ganancia CSCHD & 0,85 & 0,73 & 0,93 & 6,86 & 29 & 58 & 0,000 \\
Ganancia CSCHI & 0,91 & 0,84 & 0,95 & 11,11 & 29 & 58 & 0,000 \\
Asimetría & 0,75 & 0,54 & 0,87 & 3,95 & 29 & 58 & 0,000 \\
\hline
\end{tabular}


la familiaridad y la preparación previa que tuvieron los evaluadores con el instrumento de medición, y el aprendizaje adecuado de la técnica de medición.

\section{CONCLUSIONES}

Los valores obtenidos con la prueba v-HIT en esta investigación, por los 3 examinadores para las variables ganancia del CSCHD, ganancia del CSCHI y asimetría, se corresponden con los de estudios similares, tanto a nivel nacional como internacional, avalando los valores normativos existentes.

La prueba v-HIT mostró una alta confiabilidad interevaluador para todas las variables de estudio a través de los gráficos de Bland y Altman, donde se determinó gran concordancia entre las mediciones realizadas por las distintas parejas de evaluadores a un mismo participante; y mediante el $\mathrm{CCl}$ donde se obtuvo un alto índice de concordancia entre los 3 examinadores.

Con respecto a la determinación de la ausencia de sacadas correctivas en cada una de las medi-

\section{BIBLIOGRAFÍA}

1. Pérez N, Gallegos V, Barona L, Manrique R. Exploración clínica y videoasistida del reflejo vestíbulo-oculomotor: análisis comparativo. Acta Otorrinolaringol Esp 2012; 63: 429-35.

2. Pérez N, Eza P. Normal Gain of VOR with Refixation Saccades in Patients with Unilateral Vestibulopathy. J Int Adv Otol 2015; 11: 133-7.

3. MacDougall HG, Weber KP, McGarvie LA, Halmagy GM, CuRThoys IS. The video head impulse test: diagnostic accuracy in peripheral vestibulopathy. Neurology 2009; 73: 1134-41.

4. Carriel C, Rojas M. Prueba de impulso cefálico: Bases fisiológicas y métodos de registro del Reflejo Vestíbulo Oculomotor. Rev Otorrinolaringol Cir Cabeza Cuello 2013; 73: 206-12.

5. Weber KP, MacDougall HG, Halmagy GM, Curthoys IS. Impulsive testing of semicircular-canal function using video-oculography. Ann N Y Acad Sci 2009; 1164: 486-91. ciones realizadas por los 3 examinadores, hubo un acuerdo total. Esto se encuentra en directa relación con el estado sano de los participantes del estudio, ya que la presencia de sacadas correctivas en sujetos jóvenes y sanos, independiente de los valores de ganancia de los canales semicirculares y asimetría, implicaría una disfunción del RVO.

Los resultados de esta investigación cobran relevancia debido a que los valores de ganancia y asimetría que entrega la prueba v-HIT, son utilizados rutinariamente para realizar diagnósticos clínicos en pacientes que sufren alteraciones de equilibrio y vértigo; por lo cual, no pueden mostrar diferencias clínicamente significativas según quien realiza el examen; ya que cada centro clínico puede contar con evaluadores distintos.

Finalmente, la prueba v-HIT demostró ser una prueba confiable independiente del examinador que la realice, siempre y cuando, los evaluadores conozcan el equipo a utilizar y manejen la técnica del examen. Sin embargo, es importante que se sigan realizando estudios similares que permitan avalar los resultados obtenidos en esta investigación.

6. Breinbahuer H, Anabalón J, Aracena K, Nazal $D$, Baeza M. Experiencia en el uso videoImpulso Cefálico (v-HIT) en la evaluación del reflejo vestíbulo-ocular para canal semicircular horizontal. Rev Otorrinolaringol Cir Cabeza Cuello 2013; 73: 115-24.

7. McGarvie LA, MacDougall HG, Halmagy GM, Burguess AM, Weber KP, Curthoys IS. The Video Head Impulse Test (vHIT) of Semicircular Canal Function - Age-Dependent Normative Values of VOR Gain in Healthy Subjects. Front Neurol 2015; 6: 154.

8. Gómez A, Bruna A, Franzoy D, Julio M, Olivares R, Vicencio N. Eficiencia del Reflejo Vestíbulo Ocular mediante la aplicación de la prueba Video Head Impulse Test, en estudiantes de primer año de las Escuelas de Fonoaudiología y Tecnología Médica de la Universidad de Valparaíso. Rev Otorrinolaringol Cir Cabeza Cuello 2015; 75: 137-44.

9. Boleas-Aguirre M, Miglaccio A, Carey J. Registro del reflejo vestibulo oculomotor con la técnica de 
la bobina corneal en campo magnético (scleral search coil). Revisión de afecciones vestibulares periféricas. Acta Otorrinolaringol Esp 2007; 58: 321-6.

10. Wilches-Luna EC, Sandoval LM, López DJ. Confiabilidad intra e inter evaluador de la medición de la presión inspiratoria máxima (Pimáx) en treinta sujetos sanos de la ciudad de Cali. Rev Cienc Salud 2016; 14: 329-38.

11. Cardoso JR, Azevedo nCt, Cassano CS, Kawano MM, ÂMBAR G. Intra and inter observer reliability of angular kinematic analysis of the hip joint during the sit-and-reach test to measure hamstring length in university students. Rev Bras Fisioter 2007; 11: 133-8.

12. Cortés R, Rubio J, Gaitán H. Métodos estadísticos de evaluación de la concordancia y la reproducibilidad de pruebas diagnósticas. Rev Colomb Obstet Ginecol 2010; 61: 247-55.

13. Carrasco J, Jover Ll. Métodos estadísticos para evaluar concordancia. Med Clin (Barc) 2004; 122: 28-3.

14. Koo TK, LI MY. A Guideline of Selecting and Reporting Intraclass Correlation Coefficients for Reliability Research. J Chiropr Med 2016; 15: 155-63.

15. Bland JM, Altman DG. Statistical methods for assessing agreement between two methods of clinical measurement. Lancet 1986; i: 307-10.

16. Weber K, Aw S, Todd M, McGarvie L, Curthoys I, HaLmagyı G. Head Impulse Test in unilateral vestibular loss. Vestibulo-ocular reflex and catch-up saccades. J Neurol 2008; 70: 45463. 